\title{
Erratum
}

\section{Erratum: Wang et al., "14-3-3 Proteins Reduce Cell-to-Cell Transfer and Propagation of Pathogenic $\alpha$-Synuclein"}

In the article "14-3-3 Proteins Reduce Cell-to-Cell Transfer and Propagation of Pathogenic $\alpha$-Synuclein" by Bing Wang, Rachel Underwood, Anjali Kamath, Colleen Britain, Michael B. McFerrin, Pamela J. McLean, Laura A. Volpicelli-Daley, Robert H. Whitaker, William J. Placzek, Katelyn Becker, Jiyan Ma, and Talene A. Yacoubian, which appeared on pages 8211-8232 of the September 19, 2018 issue, Figure $13 a$ included an error in the representative images used to depict phospho-S129 $\alpha$ syn and MAP immunostaining. On re-review of the images for the wild-type and transgenic conditions, it appears that the imager (who was blinded during imaging) inadvertently reimaged the same condition twice and thus mislabeled the resulting images from which the composite was made. This error was not initially caught because reimaging of the same slide led to fainter immunofluorescence and the region of the slide imaged was shifted. This error has been corrected, and the revised Figure $13 a$ includes the correct images for the wild-type and 14-3-30 transgenic conditions. The new images are for treatment with $\alpha$ syn fibrils at $0.5 \mu \mathrm{g} / \mathrm{ml}$ instead of $1 \mu \mathrm{g} / \mathrm{ml}$. The quantifications shown in Figure 13, $c$ and $d$, were not affected. Neither the old nor the new representative images were used to quantify the area that stained positive for phospho-S129 $\alpha$ syn. The corrected figure and figure legend are presented here. Figure 13 has been corrected in the online PDF version. 


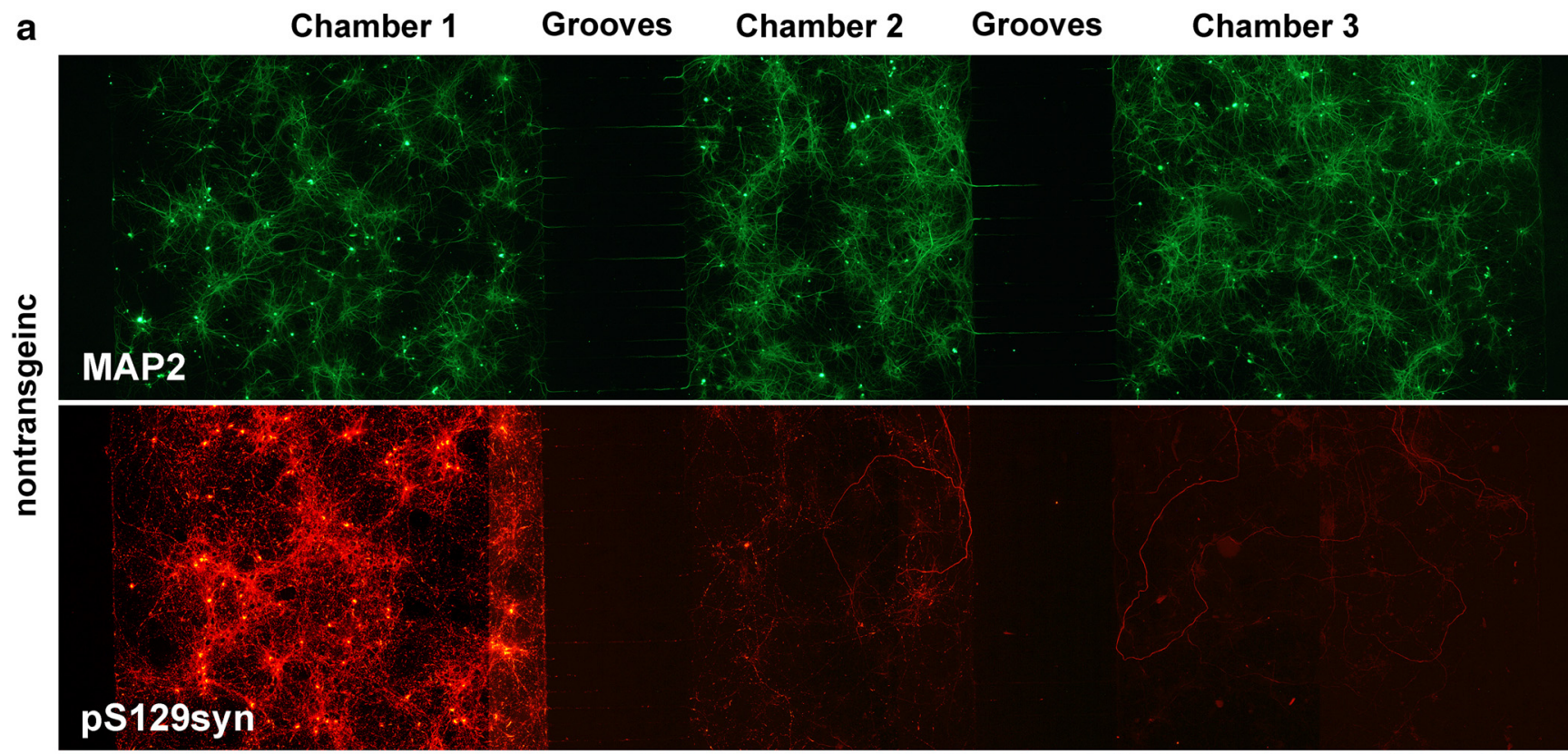

\section{}

\section{MAP2}

\section{pS129syn}

b

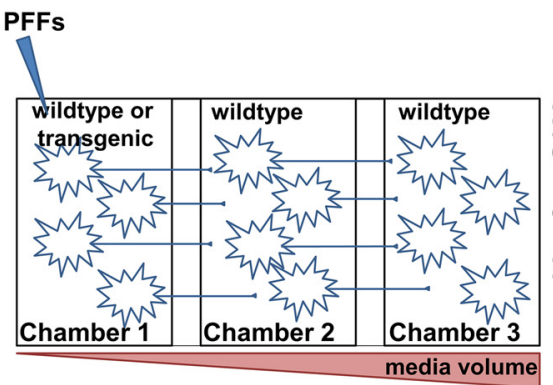

C

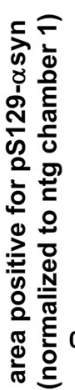

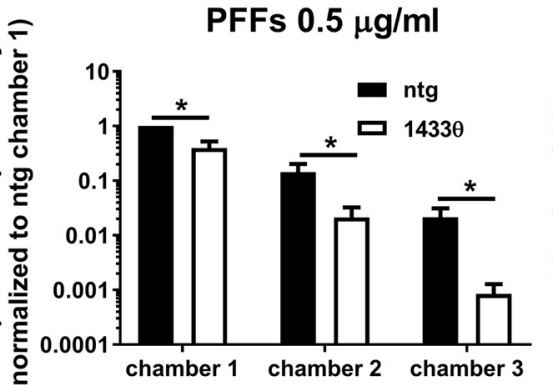

chamber 1 chamber 2 chamber 3 d

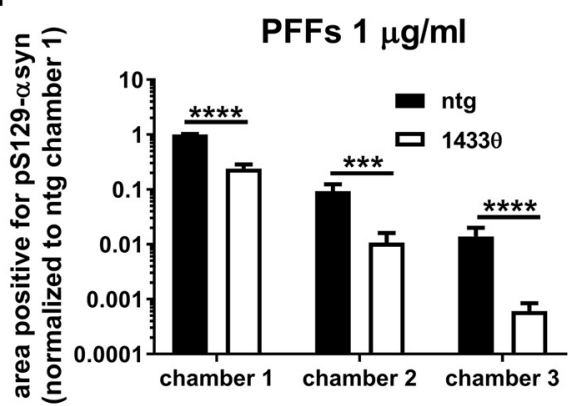

Figure 13. 14-3-3 $\theta$ reduces $\alpha$ syn spread in the PFF model. $\boldsymbol{a}$, Immunocytochemistry for pS129- $\alpha$ syn (red) and MAP2 (green) in primary neurons in multichamber, microfluidic culture devices. Primary neurons from nontransgenic or 14-3-30 mice were plated in Chamber 1, and nontransgenic mouse neurons were plated in the other chambers of multichamber, microfluidic devices. Neurons in Chamber 1 were treated with $0.5 \mu \mathrm{g} / \mathrm{ml} \mathrm{PFFs}$. $\boldsymbol{b}$, Schematic of experimental design. Neurons from nontransgenic or 14-3-3 $\theta$ mice were plated in Chamber 1 , and nontransgenic mouse neurons were plated in chambers 2 and 3. At DIV5, neurons in Chamber 1 were treated with PFFs. Spread of PFFs into the other chambers was prevented by hydrostatic pressure differences between chambers. c, Quantification of area covered by $\mathrm{pS} 129-\alpha$ syn immunoreactivity in each chamber of primary cultures from nontransgenic and 14-3-3 $\theta$ transgenic mice treated with $0.5 \mu \mathrm{g} / \mathrm{ml} \mathrm{PFFs}$. $n=4$. $\boldsymbol{d}$, Quantification of area covered by pS129- $\alpha$ syn immunoreactivity in each chamber of primary cultures from nontransgenic and 14-3-3 $\theta$ transgenic mice treated with $1 \mu \mathrm{g} / \mathrm{ml}$ PFFs. $n=$ $8 .{ }^{*} p<0.05,{ }^{* * *} p<0.001,{ }^{* * *} p<0.0001$ (Student's $t$ test, with correction for multiple comparisons using Holm-Sidak method; adjusted $p$ values shown). Error bars represent SEM. 